\title{
Comparison of Efficacy and Safety of Combination of Metformin with Tenegliptin, Metformin with Voglibose and Metformin with Glimepiride in Treatment of Patients with Type 2 Diabetes Mellitus
}

\section{Dr. Arshad Hasan ${ }^{1 *}$, Dr. Pratik ${ }^{2}$}

${ }^{1}$ Associate Professor, ${ }^{2}$ Assistant Professor, Department of Pharmacology, Rama Medical College Hospital \& Research Center, Hapur Uttar Pradesh India

DOI: $10.36348 /$ sjmps.2020.v06i04.006

| Received: 11.04.2020 | Accepted: 18.04.2020 | Published: 25.04.2020

*Corresponding author: Dr. Arshad Hasan

\section{Abstract}

Background: Metformin when used in combination with other drugs such as Tenegliptin, voglibose and glimepiride is effective and well tolerated in diabetes management. The aim of the study was to compare the efficacy and safety of combination of metformin with Tenegliptin, voglibose and glimepiride in patients with type 2 DM. Methods: In this prospective, randomized clinical trial study 150 patients were included. The patients were divided into three groups, each group comprising of 50 patients. In all the patients fasting plasma glucose, 2 hours postprandial plasma glucose, and hemoglobin A1c (HbA1c) level were assessed before starting the treatment and after completion of the treatment. Group I consisted of patients who were prescribed metformin $500 \mathrm{mg}$ with Tenegliptin $20 \mathrm{mg}$, in Group II, consisted of patients who were prescribed metformin $500 \mathrm{mg}$ with voglibose $0.2 \mathrm{mg}$, and Group III, consisted of patients who were prescribed metformin $500 \mathrm{mg}$ with glimepiride $1 \mathrm{mg}$. The outcome of the therapy was based on the level of improvement in the blood parameters. Results: There was a significant reduction of HbA1c, fasting plasma glucose and 2 hours postprandial plasma glucose levels at the start and after 12 weeks of treatment in all the groups. Intergroup comparison between both the groups revealed no significant difference in terms of glycaemic control. Conclusion: From above results it was concluded that all three groups have equal efficacy in controlling the fasting plasma glucose, 2 hours postprandial plasma glucose and HbA1c level.

Keywords: Diabetes, Glimepiride, Metformin, Tenegliptin, Voglibose.

Copyright @ 2020: This is an open-access article distributed under the terms of the Creative Commons Attribution license which permits unrestricted use, distribution, and reproduction in any medium for non-commercial use (NonCommercial, or CC-BY-NC) provided the original author and source are credited.

\section{INTRODUCTION}

Diabetes mellitus is a common chronic disease. This disease has affected an expected population of 415 million in 2015. India has become the Diabetes Capital of the World and had 69.2 million diabetic patients in 2015. This is anticipated to increase to 123.5 million in 2040[1].

Pharmacological treatment of diabetes mellitus is indicated when fasting glucose level surpasses $1600 \mathrm{mg} / \mathrm{L}$. The oral glucose-lowering drugs are utilized for management of type II diabetes mellitus. Traditionally, oral therapy is indicated in any type II diabetic in whom diet and exercise fail to accomplish satisfactory glycaemic control [2].

The oral hypoglycemic agents for Type 2 diabetes are insulin secretagogues, sulfonylureas and meglitinides, and insulin sensitizers such as biguanides and thiazolidinediones, $\alpha$-glucosidase inhibitor is voglibose, and dipeptidyl peptidase- 4 inhibitors such as Tenegliptin and glucagon-like peptide-1 agonists exist and usually prescribed [3].

For effective management of type 2 diabetes mellitus (T2DM), combination therapy, which tends to both insulin resistance and beta cell dysfunction, is important [4]. Metformin is broadly acknowledged as the first line oral agent for T2DM. When metformin alone lacks, the choice of second line treatment has remained a challenge. Notwithstanding a plenty of new agents, sulfonylureas are as yet the most acknowledged second line add-on to metformin, particularly in Indian clinical settings [1].

Tenegliptin which is a DPP-4 inhibitors is orally active and routinely prescribed as monotherapy or as an add on therapy [5]. 
Voglibose monotherapy can reduce $\mathrm{HbAlc}$ levels by $0.5 \%$ to $1.4 \%$. Dipeptidyl peptidase-4 (DPP-4) inhibitors defer the degradation of glucagon-like peptide-1 (GLP-1) and enhance endogenous GLP-1 levels, thus promoting insulin secretion [6].

Glimepiride is the only sulfonylurea affirmed by the United States Food and Drug Administration for the treatment of T2DM as monotherapy as well as in combination with metformin or insulin.

Albeit numerous oral antidiabetic agents are available, we have to pick a medication having better efficacy, less adverse effects, and lower hypoglycemic property. Hence we compared the efficacy and safety of combination of metformin with Tenegliptin; voglibose and glimepiride in patients with type $2 \mathrm{DM}$.

\section{Material ANd Methods Inclusion criteria}

Patients diagnosed with type $2 \mathrm{DM}$, both male and female of age 20-65 years, and HbA1c level below $8.5 \%$ were included in the study.

\section{Exclusion criteria}

Type 1 DM, patients with known hypersensitivity to metformin, Tenegliptin, voglibose and glimepiride, pregnant and lactating females, renal impairment, serum creatinine more than $1.4 \mathrm{mg} / \mathrm{dl}$, and significant gastrointestinal diseases were excluded from the study.

\section{Study design}

In this prospective, randomized clinical trial study 150 patients were included. The patients were divided into three groups, each group comprising of 50 patients. In all the patients haemoglobin A1c (HbA1c), fasting plasma glucose and 2 hours postprandial plasma glucose level were assessed before starting the treatment and after completion of the treatment. Group I consisted of patients who were prescribed metformin $500 \mathrm{mg}$ with Tenegliptin $20 \mathrm{mg}$, in Group II, consisted of patients who were prescribed metformin $500 \mathrm{mg}$ with voglibose $0.2 \mathrm{mg}$, and Group III, consisted of patients who were prescribed metformin $500 \mathrm{mg}$ with glimepiride $1 \mathrm{mg}$. HbA1c, FPG and PPG were monitored at baseline and subsequently every 4 weeks and at end of 12 weeks. A baseline demographic data such as age, sex, BMI, personal habits, family history, and drug history were recorded and entered in the pro forma sheet. After consultation with dietician, the patients were provided with a diet plan to follow till the next visit and were also instructed to follow physical activity. During the follow-up visit, the HbA1c, FPG and PPG were measured by collecting venous blood samples. In each follow-up visit, the patient diary was reviewed for occurrence of instances of symptoms suggestive of hypoglycemia, study drug compliance. At the end of 12 weeks of treatment, the patients were advised to attend a follow-up visit wherein the patient's HbA1c, FPG and PPG were recorded.

The outcome of the therapy was based on the level of improvement in the fasting plasma glucose (FPG), postprandial plasma glucose (PPG), and HbA1c levels.

\section{Statistical Methods}

Statistical analysis was done using the Statistical Package for the Social Sciences. Results were presented as a mean \pm standard deviation. Results on categorical measurements were presented in number (\%). Significance was assessed at 5\% level of significance. Paired Student's t-test was used to find the significance of study parameters in the three groups (intragroup analysis). Multiple comparisons were done in between groups at the end of the 3rd month using analysis of variance. Quantitative analysis was done by Chi-square test.

\section{RESULTS}

Table-1: Multiple comparisons of plasma glucose parameters in pre and post-treatment groups

\begin{tabular}{|c|c|c|c|c|c|c|c|}
\hline \multirow{2}{*}{\multicolumn{2}{|c|}{ Groups }} & \multicolumn{2}{|l|}{ HbA1c } & \multicolumn{2}{|l|}{ FPG } & \multicolumn{2}{|l|}{ PPG } \\
\hline & & Baseline & End of study & Baseline & End of study & Baseline & End of study \\
\hline $\begin{array}{l}\text { Metformin } \\
\text { With } \\
\text { Tenegliptin }\end{array}$ & $\begin{array}{l}\text { Group I } \\
(\mathrm{n}=50)\end{array}$ & $7.98 \pm 0.83$ & $6.81 \pm 0.65$ & $161.67 \pm 2.96$ & $114.95 \pm 3.25$ & $238.3 \pm 4.85$ & $158.55 \pm 5.77$ \\
\hline $\begin{array}{l}\text { Metformin } \\
\text { With } \\
\text { Voglibose }\end{array}$ & $\begin{array}{l}\text { Group II } \\
(\mathrm{n}=50)\end{array}$ & $7.55 \pm 0.56$ & $6.32 \pm 0.88$ & $165.21 \pm 3.78$ & $116.65 \pm 2.86$ & $235.6 \pm 6.62$ & $149.21 \pm 5.13$ \\
\hline $\begin{array}{l}\text { Metformin } \\
\text { With } \\
\text { Glimepiride }\end{array}$ & $\begin{array}{l}\text { Group III } \\
(n=50)\end{array}$ & $7.71 \pm 0.64$ & $6.57 \pm 0.51$ & $170.7 \pm 3.19$ & $114.8 \pm 3.53$ & $251.23 \pm 4.92$ & $164.95 \pm 4.82$ \\
\hline
\end{tabular}

There was a significant reduction of $\mathrm{HbA1c}$, fasting plasma glucose and 2 hours postprandial plasma glucose levels at the start and after 12 weeks of treatment in all the groups. Intergroup comparison between both the groups revealed no significant difference in terms of glycaemic control. 
Table-2: Adverse drug reaction

\begin{tabular}{|l|l|l|l|}
\hline $\mathbf{n = 3 5}$ & $\begin{array}{l}\text { Metformin } \\
\text { With } \\
\text { Tenegliptin }\end{array}$ & $\begin{array}{l}\text { Metformin } \\
\text { With } \\
\text { Voglibose }\end{array}$ & $\begin{array}{l}\text { Metformin } \\
\text { With } \\
\text { Glimepiride }\end{array}$ \\
\hline Headache & 1 & 0 & 2 \\
\hline Abdominal pain & 2 & 0 & 0 \\
\hline Diarrhea & 1 & 1 & 0 \\
\hline Nausea & 0 & 2 & 1 \\
\hline Vomiting & 0 & 0 & 0 \\
\hline Hypoglycemia & 1 & 0 & 3 \\
\hline
\end{tabular}

\section{DISCUSSION}

Diabetes mellitus is a chronic metabolic disorder in the metabolism of carbohydrates, proteins and lipids. It is regularly accompanied after a timeframe by specific micro vascular, macro vascular and neuropathic complications [2]. All these exude from insufficient secretion of insulin by the pancreas or inappropriate utilization of insulin by target cells [8]. The objective for diabetes management is to evade or reduce chronic diabetic complications, as well as to avoid acute problems of hyperglycemia or hypoglycemia, blindness, heart disease and limb amputation. Full-blown diabetes mellitus type II can be evaded in those with only mildly impaired glucose tolerance. Patients with type I diabetes mellitus require direct injection of insulin as their bodies cannot produce enough (or even any) insulin. For type II diabetics, diabetic management consists of a combination of diet, exercise, and weight loss, in any achievable combination depending on the patient. Patients who have poor diabetic control after lifestyle modifications are typically placed on oral hypoglycemics. Some type II diabetics fail to respond to these and must proceed to insulin therapy [2].

In the beginning time of the disease, the pancreas is able to overcome insulin resistance by producing more insulin [1]. As the disease advances, the pancreas is unable to overcome the insulin resistance; leading to the development of type $2 \mathrm{DM}$. Metformin decreases the blood glucose levels by bringing down hepatic glucose production and expanding the peripheral utilization of glucose. Metformin has regulatory actions on lipid metabolism, improves endothelial function, decreases hypercoagulation, and has a defensive effect on the cardiovascular system. Since insulin resistance is the most widespread pathology in Type 2 diabetes, metformin is the most commonly used drug to treat Type 2 diabetes along with glimepiride. ADA and EASD also recommend metformin as the first-line drug in type 2 DM [9]. Hence, in our study; we have taken metformin as the primary drug.

The present study compared the efficacy and safety of combination of metformin with Tenegliptin; voglibose and glimepiride in patients with type $2 \mathrm{DM}$.
In our study, 50 patients were taken in each group. The mean age in metformin with Tenegliptin (Group I) was 55.8 years, metformin with voglibose (Group II) was 50.44 years, and metformin with glimepiride (Group III) was 58.52 years. In group I there were 32 males and 18 females, in group II there were 36 males and 14 females, in group III there were 28 males and 22 females. Our findings are in accordance with the findings of Devarajan TV et al. [1] who studied the efficacy of combination of metformin with Glimepiride and sitagliptin with mean age of patients as 50.3 years and 48.7 years. In our study there was significant difference between levels of $\mathrm{HbA} 1 \mathrm{c}$, fasting plasma glucose and 2 hours postprandial plasma glucose when they were compared at baseline that is start of treatment and at the ends of 12 weeks. Results in our study are in accordance with the studies done by Srivastava et al. [10] who in their study of 50 patients who on metformin monotherapy reported a significant reduction in $\mathrm{HbA} 1 \mathrm{c}, \mathrm{FBG}$ and $2 \mathrm{HPPG}$ values with sitagliptin addition, when compared to baseline values.

Lim et al. [11] reported in their study that early initial combination therapy of sitagliptin and metformin in drug-naive Type 2 diabetic patients with low $\beta$-cell function has produced a significant reduction in FPG, PPG, and HbA1c (13\%) at 12 weeks.

Jeon et al. [12] reported in their study that there was a well comparable statistically significant reduction of FPG, PPG, and $\mathrm{HbA1c}$ seen in vildagliptin-metformin and glimepiride-metformin groups.

Ismail et al. [13] demonstrated that voglibose showed a significant reduction of FPG, PPG, and HbA1c level.

Devarajan TV et al. [1] have reported mild hypoglycemia among metformin with gliptin group and metformin with glimepiride group whereas abdominal discomfort and bloating were observed in metformin with voglibose group. In this study, hypoglycemia was seen in one patient in Groups I and 3 patients in group III. Other adverse drug reactions were headache, abdominal pain, diarrhea, nausea, vomiting that is illustrated in table 2 . 


\section{CONCLUSION}

It was concluded that all three groups have equal efficacy in controlling the fasting plasma glucose, 2 hours postprandial plasma glucose and $\mathrm{HbA} 1 \mathrm{c}$ level.

\section{REFERENCES}

1. Devarajan, T.V., Venkataraman, S., Kandasamy, N., Oomman, A., Boorugu, H.K., Karuppiah, S. (2017). Comparative evaluation of safety and efficacy of glimepiride and sitagliptin in combination with metformin in patients with type 2 diabetes mellitus: Indian multicentric randomized trial - START Study. Indian J Endocr Metab, 21:745-50.

2. Piero, N.M., Murugi, N.J., Mwiti, K.C., Mwenda, M.P. (2012). Pharmacological Management of Diabetes Mellitus. Asian Journal of Biochemical and Pharmaceutical Research, 2(2):375-81.

3. Kala, P., Rani, J., Kumar, J.S. (2017). A comparative study of efficacy and safety among metformin with sitagliptin, metformin with voglibose, and metformin with glimepiride in patients with type 2 diabetes mellitus. Asian $J$ Pharm Clin Res, 10(12):313-16.

4. LaSalle, J.R., Cross, L.B. (2006). Oral combination therapy with thiazolidinediones in type 2 diabetes. Is J Manag Care, 12:S369- 81?

5. Sharma, M., Sonawane, R., Marko, J.L. (2016). Comparative study of efficacy and safety of sitagliptin versus glimepiride in patients of type-2 diabetes mellitus inadequately controlled with metformin alone. Int $J$ Adv Med, 3:564-8.

6. Shi, C., Zhang, R., Bai, R., Liu, D., Wang, Y., Zhang, X. (2019). Efficacy and safety of sitagliptin added to metformin and insulin compared with voglibose in patients with newly diagnosed type 2 diabetes. CLINICS, 74:e736.
7. Basit, A., Riaz, M., Fawwad, A. (2012). Glimepiride: Evidence- based facts, trends, and observations. Vasc Health Risk Manag; 8:463- 72.

8. Piero, N. M., Njagi, M. J., Kibiti, M. C., Ngeranwa, J. J. N., Njagi, N. E., Njue, W. M., \& Gathumbi, P. K. (2012). Herbal management of diabetes mellitus: a rapidly expanding research avenue. International Journal of Current Pharmaceutical Research, 4(2), 1-4.

9. Yada, N. I. K. H. I. L., Thrulapati, D. T., Maheshwari, A. R. P. I. T., Upadhyay, V. I. H. A. R., \& Shah, P. R. A. N. I. T. (2015). A study on the prescribing pattern of antidiabetic drugs in a community clinic in Telangana state. Int J Pharm Pharm Sci, 7(9), 222-6.

10. Srivastava, S., Saxena, G. N., Keshwani, P., \& Gupta, R. (2012). Comparing the efficacy and safety profile of sitagliptin versus glimepiride in patients of type 2 diabetes mellitus inadequately controlled with metformin alone. $J$ Assoc Physicians India, 60(2), 27-30.

11. Lim, S., An, J. H., Shin, H., Khang, A. R., Lee, Y., Ahn, H. Y., \& Park, K. S. (2012). Factors predicting therapeutic efficacy of combination treatment with sitagliptin and metformin in type 2 diabetic patients: the COSMETIC study. Clinical endocrinology, 77(2), 215-223.

12. Jeon, H. J., \& Oh, T. K. (2011). Comparison of vildagliptin-metformin and glimepiride-metformin treatments in type 2 diabetic patients. Diabetes \& metabolism journal, 35(5), 529-535.

13. Ismail, D. T. S. E. S., \& Deshmukh, D. S. A. (2012). Comparative study of effect of alpha glucosidase inhibitors-Miglitol, acarbose and voglibose on postprandial Hyperglycemia and glycosylated hemoglobin in type-2 Diabetes Mellitus. Diabetes, 7, 8. 